\title{
Calibración del modelo predictivo de accidentes de tránsito del HSM en carreteras del cantón Loja (Ecuador)
}

\section{Calibration of the highway safety manual accident prediction model of HSM in two lane roads in Loja canton (Ecuador)}

\author{
Yasmany García-Ramírez \\ Pamela Rojas \\ Edwin Duque \\ Henrry Rojas-Asuero \\ Universidad Técnica Particular de Loja, Ecuador \\ Autor para correspondencia: ydgarcia1@utpl.edu.ec, pamelarojascazar@hotmail.com, \\ epduque@utpl.edu.ec,hvrojas@utpl.edu.ec \\ Fecha de recepción: 26 de enero de 2018 - Fecha de aceptación: 25 de julio de 2018
}

Resumen: El modelo de predicción de accidentes de tránsito del Manual de Seguridad de Carreteras (HSM por sus siglas en inglés) es una herramienta que permite justificar el diseño de una carretera, sin embargo, antes de usarlo, éste debe ser calibrado de acuerdo a las condiciones locales. En ese escenario, el presente trabajo tuvo por objetivo estimar el factor de calibración del módulo de predicción de accidentes del HSM en carreteras principales del cantón Loja. Para ello, en primer lugar, se seleccionaron los tramos de estudio, para luego recolectar información histórica de los accidentes de tránsito y de las variables geométricas y de operación de cada tramo. Con la aplicación del modelo de predicción se obtuvieron los factores de calibración para carreteras de dos carriles en zonas rurales y suburbanas. Las primeras obtuvieron factores entre 0,12 a 0,25 , lo cual indica que el modelo subestima el número de accidentes registrados en esas vías, mientras, que las carreteras sub-urbanas estuvieron entre 0,00 a 3,24, lo cual muestra que en algunos casos el modelo subestima los datos reales y en otros los sobreestima; lo cual sugiere la obligatoriedad de la calibración del modelo en cualquiera de estos casos. Además, de estos factores de calibración, este trabajo aporta con el detalle de la metodología usada en la calibración del modelo de predicción de accidentes del HSM para zonas rurales y suburbanas.

Palabras Claves: calibración; modelo predictivo de accidentes del HSM; carreteras de dos carriles; urbana y sub-urbana; Loja (Ecuador)

Abstract: Accident predictive model of the Highway Safety Manual is a tool that helps justifying the design of a road, however, before using it; it must be calibrated according to local conditions. In this scenario, the aim of this work was to estimate a calibration factor of HSM accident predictive model in the main roads of Loja canton. To do this, firstly, study sections were selected, then historical traffic information and geometric and operational variables of each section were collected. Using accident predictive model, the calibration factors for two-lane roads in rural and suburban areas were estimated. The calibration factors in two-lane rural roads were between 0.12 to 0.25 , which indicates that the model underestimates the number of accidents recorder in these roads, while the two-lane sub-urban roads were between 0.00 to 3.24 , which shows that in in some cases the model underestimates the real data and in others it overestimates it; which suggests that calibration is mandatory in any of these cases. 
In addition, to the calibration factors, this work provides detailed methodology, used in the calibration of accident prediction models of the HSM for rural and suburban areas.

Key Words: calibration; accident predictive model of HSM; two-lane roads; urban and suburban; Loja (Ecuador)

\section{Introducción}

En general, el costo de construcción y mantenimiento de carretera, ya sea proyecto nuevo o rediseño, es uno de los elementos más importantes en la toma de decisiones. En algunos casos, también se incluyen los costos vehiculares (combustible, neumáticos, aceites, etc.) y costos de los usuarios (tiempo de demora), sin embargo, no es común incluir los costos por accidentes de tránsito probables en esas vías, lo cual es limitante, dado que las carreteras también deben brindar un nivel de seguridad adecuado. Ante esta necesidad se elaboró el Manual de Seguridad de Carreteras (AASHTO, 2010) con el fin proponer modelos que permitan predecir accidentes de tránsito en las vías (frecuencia y gravedad), el cual ha sido ampliamente aplicado en varias regiones a nivel mundial. El HSM (AASHTO, 2010) es aplicable para carreteras rurales de dos carriles y rurales multicarril, además también para vías arteriales, urbanas y suburbanas y debe ser calibrado para las condiciones locales, dado que sin eso, el modelo puede sobreestimar o subestimar el número de accidentes de tránsito. En todos los casos, los modelos de predicción consideran un período de tiempo, volumen del tránsito y la geometría y operación de la carretera. Cada modelo inicia con una predicción que toma en cuenta ciertas condiciones base, y que luego son modificadas por los factores de modificación de accidentes y por el factor de calibración.

En ese escenario, esta investigación tiene por objetivo calibrar el modelo de predicción de accidentes del HSM (AASHTO, 2010) para carreteras rurales de dos carriles y suburbanas de las cuatro vías principales del cantón Loja. Para exponer los resultados de esta investigación, en primer lugar, se describen las principales ecuaciones que intervienen en el modelo predictivo de accidentes de tránsito para carreteras rurales y suburbanas. Posteriormente, en la sección de materiales y métodos, se describe la obtención de geometría, las estadísticas de accidentes de tránsito y la estimación del tránsito. Luego, en la sección de resultados se muestra los resultados de los factores de calibración de las carreteras rurales y urbanas de las vías en estudio. También se discute los resultados antes de presentar las principales conclusiones del estudio.

\section{Modelo predictivo de accidentes de tránsito para carreteras rurales de dos carriles del HSM (AASHTO, 2010)}

En número de accidentes previsto de una carretera rural de dos carriles en un año determinado $\left(\mathrm{N}_{\mathrm{rs}}\right)$ se obtiene mediante el producto del número previstos de accidentes para las condiciones base $\left(\mathrm{N}_{\mathrm{spf}-\mathrm{r}}\right)$ por los factores de modificación de accidentes $\left(\mathrm{CMF}_{\mathrm{nr}}\right)$ y el factor de calibración para ajustarse a las condiciones locales $\left(\mathrm{C}_{\mathrm{r}}\right)$, tal como se muestra en la siguiente ecuación:

$$
N_{r s}=C_{r} N_{s p f-r s}\left(C M F_{1 r} * \ldots * C M F_{n r}\right)
$$

Cuando las características geométricas y de operación de la carretera son las mismas que las condiciones base, los factores de modificación de accidentes son igual a 1, caso contrario pueden éstos ser mayores a uno, cuando la característica ayuda al incremento de accidentes, y éstos son menores a uno, cuando la característica favorece la reducción de accidentes de tránsito. 
Dentro de la ecuación anterior, el número previsto de accidentes de tránsito para las condiciones base se obtienen mediante la expresión:

$$
N_{p f-r s}=T M D A * L * 365 * 10^{-6} * e^{-0,312}
$$

Donde:

TMDA: tránsito promedio diario anual (veh/día),

L: Longitud del segmento de la carretera (millas), y,

365: valor usado para determinar una estimación anual.

La ecuación anterior es aplicable para TMDA hasta 17800 veh/dia. Las condiciones base son: 12 pies para el ancho del carril, 6 pies para el ancho del espaldón, espaldón pavimentado, 3 como índice de peligrosidad de zonas laterales, 5 accesos por milla, sin curvatura horizontal o vertical, sin banda sonora central, sin carril de adelantamiento o de giro a la izquierda, sin iluminación, sin sistema de ayuda automática de la velocidad y pendiente longitudinal plana.

Los factores de modificación de accidentes $(\mathrm{CMF})$ para carreteras rurales de dos carriles son 12. Mayor detalle en su estimación puede ser observada en el Manual de Seguridad Vial (AASHTO, 2010). Esos CMFs se muestran a continuación: $\mathrm{CMF}_{1 \mathrm{r}}$ : ancho de carril, $\mathrm{CMF}_{2 \mathrm{r}}$ : ancho y tipo de espaldón, $\mathrm{CMF}_{3 \mathrm{r}}$ : curvatura horizontal, $\mathrm{CMF}_{4 \mathrm{r}}$ : peralte, $\mathrm{CMF}_{5 \mathrm{r}}$ : pendiente longitudinal, $\mathrm{CMF}_{6 \mathrm{r}}$ : densidad de accesos, $\mathrm{CMF}_{7 \mathrm{r}}$ : banda sonora central, $\mathrm{CMF}_{8 \mathrm{r}}$ : carriles de adelantamiento, $\mathrm{CMF}_{9 \mathrm{r}}$ : carriles de giro a la izquierda, $\mathrm{CMF}_{10 \mathrm{r}}$ : zonas laterales de la carretera, $\mathrm{CMF}_{11 \mathrm{r}}$ : iluminación, y $\mathrm{CMF}_{12 \mathrm{r}}$ : sistema automático de control de la velocidad.

Por otra parte, el factor de calibración se obtiene de la relación entre la sumatoria del número de accidentes observados en la carretera dividido para la sumatoria del número de accidentes previstos con el modelo para un mismo periodo de tiempo, tal como se muestra en la siguiente ecuación:

$$
c_{r}=\frac{\sum N_{o b s}}{\sum N_{p r e}}
$$

Este factor de calibración ha sido obtenido varios investigadores, por ejemplo: 0,17 (Martinelli, La Torre, \& Vadi, 2009), 0,74 (Xie, Gladhill, Dixon, \& Monsere, 2011), 1,47 (Lubliner, 2011), 1,40 (Williamson \& H., 2012), 0,19 (García \& Altamira, 2012a), 0,15 (García \& Altamira, 2012b), 0,82 (Sun, Brown, Edara, Carlos, \& Nam, 2013), 0,85 (Berardo, Freire, Marchesini, Tartabini, \& Vanoli, 2013), 1,50 (Berardo, 2015). En estos casos, la mayoría de los factores de calibración son menores a 1, lo que significa que el modelo de predicción de accidentes del HSM (AASHTO, 2010) sobreestima el número de accidentes reales. Sólo en tres casos, reportados en Estados Unidos de América (Lubliner, 2011; Williamson \& H., 2012) y Brasil (Berardo, 2015) tuvieron valores mayores a 1, lo que significa que el modelo del HSM (AASHTO, 2010) subestima el número de accidentes reales en esas carreteras.

\section{Modelo predictivo de accidentes de tránsito para carreteras suburbanas de dos carriles del HSM (AASHTO, 2010) \\ El módulo predictivo de accidentes de tránsito en el área suburbana toma en cuenta los accidentes ocasionados por los vehículos, bicicletas, peatones y entre ellos, excepto los choques entre bicicletas y peatones. Mayores detalles del cálculo se puede observar en el capítulo 12 del}


Manual de Seguridad Vial (AASHTO, 2010). El número de accidentes previsto para carreteras suburbanas de dos carriles se calcula con la siguiente ecuación:

$$
N_{r s}=C_{r}\left(N_{b r}+N_{p e d r}+N_{b i k e r}\right)
$$

Donde:

$\mathrm{N}_{\mathrm{rs}}$ : número de accidentes previsto para el segmento de carretera considerado,

$\mathrm{C}_{\mathrm{r}}$ : factor de calibración para ajustarse a las condiciones locales,

$\mathrm{N}_{\text {br: }}$ número de accidentes previstos para las condiciones base después de la aplicación de factores de modificación de accidente, excepto vehículo-peatón y vehículo-bicicleta,

$\mathrm{N}_{\text {pedr: }}$ número de accidentes previsto entre vehículo-peatón,

$\mathrm{N}_{\text {biker: }}$ número de accidentes previstos entre vehículo-bicicleta.

El número de accidentes previsto para las condiciones base (Nbr) se calcula con la siguiente expresión:

$$
N_{r s}=N_{s p f-r s}\left(C M F_{1 r} * \ldots * C M F_{n r}\right)
$$

Donde:

$\mathrm{N}_{\text {spf-rs: }}$ número de accidentes previstos para las condiciones base, $\mathrm{CMF}_{\mathrm{nr}}$ : factores de modificación de accidentes.

Adicionalmente, $\mathrm{N}_{\text {spf-rs }}$ se calcula con la siguiente expresión:

$$
N_{s p f-r s}=N_{b r m v}+N_{b r s v}+N_{b r d w y}
$$

Donde:

$\mathrm{N}_{\text {brmv: }}$ : número de accidentes previstos entre varios vehículos para las condiciones base, $\mathrm{N}_{\text {brsvr: }}$ número de accidentes previstos que involucran a un solo vehículo para las condiciones base, $y$,

$\mathrm{N}_{\text {brdwy: }}$ número de accidentes previstos entra varios vehículos relacionados con la vía para las condiciones base.

Asimismo, el $\mathrm{N}_{\text {brmv }}, \mathrm{N}_{\text {brsvr }} \mathrm{y} \mathrm{N}_{\text {brdwy }}$ para dos carriles no divididos (2U) se calcula con las ecuaciones:

$$
\begin{gathered}
N_{b r m v}=e^{(-15,22+1,68 * \operatorname{Ln}(T M D A)+\operatorname{Ln}(L))} \\
N_{b r s v}=e^{(-5,47+0,56 * \operatorname{Ln}(T M D A)+\operatorname{Ln}(L))} \\
N_{b r d w y}=\sum\left(2 N_{j}\left(\frac{T M D A}{15000}\right)^{t}\right)
\end{gathered}
$$

Donde:

TMDA: tránsito promedio diario anual (veh/día),

L: longitud del segmento (millas), y,

$\mathrm{N}_{\mathrm{j}}$ : varía en función del tipo de vía (mayor comercial: 0,158, menor comercial: 0,050, mayor industrial/institucional: 0,172, menor industrial/institucional: 0,023, mayor residencial: 0,083, menor residencial: 0,016, otro: 0,025).

Las condiciones base para segmentos suburbanos son: no tiene estacionamiento en la calle, no hay objetos fijos en el borde la carretera, el ancho de la mediana es de 15 pies, no tiene iluminación y tampoco tiene un sistema de ayuda automático de la velocidad. 
Los factores de modificación de accidentes $(\mathrm{CMF})$ para el $\mathrm{N}_{\mathrm{br}}$ son los siguientes: $\mathrm{CMF}_{1 \mathrm{r}}$ : estacionamiento en la calle, $\mathrm{CMF}_{2 \mathrm{r}}$ : objetos fijos en las zonas laterales de la carretera, $\mathrm{CMF}_{3 \mathrm{r}}$ : ancho de la mediana, $\mathrm{CMF}_{4 \mathrm{r}}$ : iluminación, y $\mathrm{CMF}_{5 \mathrm{r}}$ : sistema automático de control de la velocidad. Por otro lado, el número de accidentes vehículo-peatón (Npedr) para dos carriles de un segmento de la carretera se determina usando la ecuación:

$$
N_{p e d r}=N_{b r} * f_{p e d r}
$$

Donde:

$\mathrm{f}_{\text {pedr: }}$ factor de ajuste de accidentes peatonales $(0,036$ para velocidad límite de $48 \mathrm{~km} / \mathrm{h}$ o menos y 0,005 para velocidades límite mayores a $48 \mathrm{~km} / \mathrm{h}$ ).

Por otra parte, el número de accidentes entre vehículos y ciclistas en carreteras de dos carriles en un segmento se calcula por:

Donde:

$$
N_{\text {biker }}=N_{b r} * f_{\text {biker }}
$$

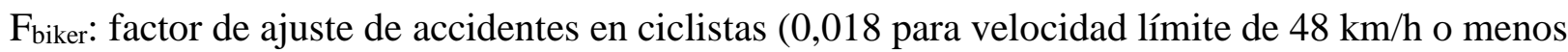
y 0,004 para velocidades límite mayores a $48 \mathrm{~km} / \mathrm{h}$ ).

Los factores de calibración de carreteras suburbanas se obtienen se igual forma que los factores de calibración de carreteras rurales, es decir, relacionando el número de accidentes observados y el número de accidentes observados con el modelo del HSM (AASHTO, 2010). Los estudios de calibración del modelo predictivo de accidentes de tránsito en carreteras suburbanas han sido escasos. El único encontrado fue el realizado en Missouri con un valor de 0,84 (Sun et al., 2013). En este caso, el modelo predictivo del HSM (AASHTO, 2010) sobredimensiona el número de accidentes reales que ocurrieron en esas carreteras.

\section{Métodos}

Para aplicar el método predictivo del HSM (AASHTO, 2010) es necesario contar con la geometría de la carretera, estadísticas de accidentes de tránsito y la estimación del tránsito, lo cual se describe en esta sección.

\section{Obtención de la geometría de la carretera}

La geometría de la carretera comprende el alineamiento horizontal, vertical y sección transversal. El alineamiento horizontal fue principalmente determinado mediante imágenes satelitales (Autodesk, 2015; Bing, 2015), mientras que el alineamiento vertical (pendientes) fue estimada mediante el uso de equipos con GPS. Asimismo, la sección transversal (ancho de carril, ancho de espaldón) fue determinada mediante mediciones en campo. Este procedimiento fue aplicado para todas las carreteras, excepto para la vía Loja-Catamayo, considerando de que se contó con el estudio proyecto de esa carretera (MTOP \& Asociación del Sur, 2015). Otras características de las carreteras como iluminación, presencia de banda sonora, presencia de carril de adelantamiento, entre otras, fueron determinadas mediante recorridos vehiculares o con el uso del Street View (Google, 2015). Algunos resultados de estos cálculos se pueden ver en la Tabla 1 y 2. La Tabla 1 contiene los tramos rurales de las carreteras en estudio, mientras que la Tabla 2 contiene los tramos suburbanos.

Tabla 1. Características de los tramos rurales del estudio 


\begin{tabular}{|c|c|c|c|c|c|c|}
\hline $\begin{array}{l}\text { Carretera } \\
\text { (código) }\end{array}$ & $\begin{array}{l}\text { Longitud } \\
\text { de tramo } \\
(\mathbf{k m})\end{array}$ & $\begin{array}{r}\text { Ancho } \\
\text { de carril } \\
\text { (m) }\end{array}$ & $\begin{array}{l}\text { Ancho de } \\
\text { espaldón } \\
\text { (m) }\end{array}$ & $\begin{array}{c}\text { Pendiente } \\
\text { longitudinal } \\
(\%)\end{array}$ & $\begin{array}{l}\text { Accesos } \\
(\mathbf{u} / \mathbf{k m})\end{array}$ & $\begin{array}{c}\text { Índice de } \\
\text { peligrosidad }\end{array}$ \\
\hline $\begin{array}{c}\text { Loja - } \\
\text { Catamayo } \\
(\text { E35/E50) }\end{array}$ & 29,21 & 3,65 & 1,20 & $0,44-7,00$ & $0-1,16$ & $3-7$ \\
\hline $\begin{array}{l}\text { Loja - Oña } \\
\text { (E35) }\end{array}$ & 92,89 & 3,65 & 1,20 & $0,07-6,81$ & $0-1,16$ & $3-6$ \\
\hline $\begin{array}{c}\text { Loja - } \\
\text { Sabanilla } \\
(\text { E50) }\end{array}$ & 9,62 & 3,65 & 0,30 & $3,08-8,71$ & $0-0,38$ & $4-6$ \\
\hline $\begin{array}{c}\text { Loja - } \\
\text { Malacatos } \\
(\text { E682) }\end{array}$ & 19,11 & 3,65 & 0,60 & $4,90-10,00$ & $0-0,39$ & $4-7$ \\
\hline
\end{tabular}

Todos los tramos rurales de medición atravesaron terreno montañoso o escarpado. Estas carreteras no tuvieron franjas sonoras, las cuales puede ayudar a reducir accidentes de tránsito por somnolencia. Tampoco tuvieron carriles de adelantamiento, lo cual reduce la posibilidad de accidente frontal, ni tampoco tienen sistemas automáticos de control de velocidad, lo que al limitar la velocidad, también puede reducir la frecuencia de accidentes, sobretodo la gravedad del accidente. Asimismo, cabe aclarar que las carreteras de prueba no tuvieron iluminación, a excepción de una pequeña parte al llegar o salir de la ciudad de Loja. Otros detalles de estos tramos se pueden ver en la Tabla 1. En esta tabla se pueden ver las diferencias entre carreteras con respecto a la longitud del tramo, ancho del espaldón, pendiente longitudinal, accesos por kilómetro y el índice de peligrosidad.

Los tramos suburbanos se encuentran en las mismas carreteras rurales descritas anteriormente. Todo este tramo tiene iluminación en casi toda su longitud, no tienen estacionamientos laterales y su distancia a la que están ubicados los objetos laterales es de 1,50 $\mathrm{m}$. El ancho del carril y ancho de espaldón fueron similares a los de la Tabla 1. Mayores detalles se pueden ver en la Tabla 2.

Tabla 2. Características de los tramos suburbanos del estudio

\begin{tabular}{lccc}
\hline Carretera (código) & Descripción del tramo suburbano & $\begin{array}{c}\text { Longitud } \\
(\mathbf{k m})\end{array}$ & Tipo de zona \\
\hline Loja - Catamayo (E35/E50) & Salida de Loja & 3,61 & Residencial \\
& Llegada a Catamayo & 2,46 & Residencial \\
Loja - Oña (E35) & Salida de Loja & 4,57 & Otras \\
& Santiago & 0,87 & Otras \\
& San Lucas & 1,46 & Residencial \\
& Saraguro & 2,53 & Otras \\
Loja - Sabanilla (E50) & Salida de Loja & 3,75 & Otras \\
Loja - Malacatos (E682) & Salida de Loja & 5,41 & Residencial \\
& & & Industrial \\
& Rumishitana & 0,64 & Otras \\
& Landangui & 1,63 & Otras \\
& Llegada a Malacatos & 1,23 & Otras \\
\hline
\end{tabular}

\section{Accidentes de tránsito}

Las estadísticas de accidentes de tránsito se obtuvieron de la base de datos de la Policía Nacional para los años 2014, 2015 (Policía Nacional, 2017). De esta base de datos, se obtuvo el 
número de accidentes de tránsito registrados para los tramos rurales y suburbanos para los tres años en consideración. En los tramos rurales, el número de accidentes de tránsito estuvo de acuerdo al siguiente detalle: Loja - Catamayo: 28 accidentes, Loja - Oña: 26 accidentes, Loja Sabanilla: 8 accidentes y Loja - Malacatos: 15 accidentes. Por otro lado, en los tramos suburbanos se registraron accidentes de tránsito en esos mismos años según el siguiente detalle: Salida a Loja (Loja-Catamayo): 6 accidentes, Llegada a Catamayo (Loja- Catamayo): 1 accidente, Salida de Loja (Loja-Oña): 6 accidentes, Santiago (Loja-Oña): 0 accidentes, San Lucas (Loja-Oña): 0 accidentes y Saraguro (Loja-Oña): 4 accidentes, Salida de Loja (LojaSabanilla): 8 accidentes, Salida de Loja (Loja-Malacatos): 8 accidentes, Rumishitana (LojaMalacatos): 1 accidente, Landangui (Loja-Malacatos): 3 accidentes, Llegada a Malacatos (LojaMalacatos): 0 accidentes..

\section{Estimación del tránsito}

El tránsito promedio diario anual (TMDA) es una variable muy influyente en la calibración del modelo de accidentes, el cual debe ser de los mismos años en consideración. El tránsito promedio diario del año 2016 fue establecido con mediciones con tubos piezométricos obtenidos previamente por otras investigaciones (Ortiz, 2017; Zúñiga, 2017). TMDA para años 2014 y 2015 fueron estimados a partir el TMDA del 2016, y usando la ecuación del crecimiento vehicular con una tasa estimada por el Ministerio de Transporte y Obras Públicas (MTOP, 2012). Esta metodología ya fue usada por otro investigador (Berardo, 2015). El resultado de la estimación del tránsito se puede ver en la Tabla 3.

Tabla 3. Tránsito promedio diario anual (TMDA) para las carreteras del estudio

\begin{tabular}{cccc}
\hline Carretera (código) & \multicolumn{3}{c}{ TMDA (veh/día) } \\
\cline { 2 - 4 } & 2014 & 2015 & 2016 \\
Loja - Catamayo (E35/E50) & 3381 & 3533 & 3708 \\
Loja - Oña (E35) & 2155 & 2252 & 2363 \\
Loja - Sabanilla (E50) & 1714 & 1791 & 1879 \\
Loja - Malacatos (E682) & 2614 & 2732 & 2867 \\
\hline
\end{tabular}

\section{Resultados}

En esta sección se detallan los resultados obtenidos del factor de calibración en tramos rurales y suburbanos. Los resultados de la calibración del modelo en los tramos rurales se pueden ver en la Tabla 4. En esta tabla se puede observar, en todos los casos, que el modelo de predicción del HSM (AASHTO, 2010) sobreestima el número de accidentes de tránsito, dado que los accidentes previstos para esos tres años son mucho mayores que los accidentes observados o registrados en esos tres años. Cabe mencionar que los sitios de medición fueron superiores a los solicitados en el HSM (AASHTO, 2010), por lo que, los estos resultados podrían tener una mayor confiabilidad.

Tabla 4. Factores de calibración (fc) para los tramos rurales de las vías en estudio

\begin{tabular}{ccccc}
\hline Carretera (código) & Sitios & $\begin{array}{l}\text { Accidentes } \\
\text { previstos }\end{array}$ & $\begin{array}{c}\text { Accidentes } \\
\text { observados }\end{array}$ & fc \\
\hline Loja-Catamayo & 214 & 114,00 & 28 & 0,25 \\
(E35/E50) & & & & \\
Loja - Oña (E35) & 694 & 226,53 & 26 & 0,12 \\
Loja - Sabanilla (E50) & 113 & 38,32 & 8 & 0,21 \\
Loja- Malacatos (E682) & 168 & 76,52 & 15 & 0,20 \\
\hline
\end{tabular}


Por otro lado, los resultados de calibración de los tramos suburbanos se pueden ver en la Tabla 5. En esta tabla, existen tramos en donde el modelo de predicción de accidentes sobreestima el número de accidentes, sin embargo, en la mayoría de casos, el modelo los subestima. Esto posiblemente se deba a que en un ambiente suburbano existen mayores interacciones entre los usuarios viales, por ejemplo, más vehículos, más peatones, más objetos fijos, en fin, más información que el conductor debe procesar.

\begin{tabular}{ccccc}
\multicolumn{6}{c}{ Tabla 5. Factores de calibración (fc) } & para los tramos suburbanos de las vías en estudio \\
\hline $\begin{array}{c}\text { Sitio de la carretera } \\
\text { (código) }\end{array}$ & Sitios & $\begin{array}{l}\text { Accidentes } \\
\text { previstos }\end{array}$ & $\begin{array}{c}\text { Accidentes } \\
\text { observados }\end{array}$ & fc \\
\hline $\begin{array}{c}\text { Salida de Loja (E35/E50) } \\
\text { Llegada a Catamayo }\end{array}$ & 19 & 4,17 & 6,00 & 1,44 \\
$\quad 15$ & 3,00 & 1,00 & 0,33 \\
(E35/E50) & & & & \\
Salida de Loja (E35) & 32 & 3,68 & 6,00 & 1,63 \\
Santiago (E35) & 3 & 0,68 & 0,00 & 0,00 \\
San Lucas (E35) & 25 & 1,14 & 0,00 & 0,00 \\
Saraguro (E35) & 15 & 1,99 & 4,00 & 2,01 \\
Salida de Loja (E50) & 38 & 2,47 & 8,00 & 3,24 \\
Salida de Loja (E682) & 49 & 5,16 & 8,00 & 1,55 \\
Rumishitana (E682) & 9 & 0,56 & 1,00 & 1,79 \\
Landangui (E682) & 11 & 1,83 & 3,00 & 1,64 \\
Llegada a Malacatos (E682) & 2 & 1,13 & 0,00 & 0,00 \\
\hline
\end{tabular}

En ambos casos, los resultados refuerzan la idea de que para usar el modelo de predicción de accidentes de tránsito del HSM (AASHTO, 2010) es obligatoria su calibración, en caso de no poder hacerlo (por ejemplo, debido a la falta de datos), se puede adoptar un coeficiente de calibración de otra vía que tenga similares características geométricas y de operación.

\section{Discusión}

La mayoría de las características geométricas de las carreteras fueron tomadas de manera indirecta, mediante imágenes satelitales o recorridos virtuales. Cabe aclarar que esta metodología no fue un propuesta original de este estudio, ya que fue usada y probada por otro investigador (Berardo, 2015). La ventaja de este estudio comparada con el de Berardo (2015), es que, en este caso, se tuvo acceso a los planos de la vía Loja-Catamayo, los cuales fueron levantados por medios topográficos tradicionales, por lo tanto, fueron de mayor precisión. Entonces, se desarrolló una metodología para obtener la geometría con las imágenes satelitales, la cual fue corregida con la ayuda de los planos topográficos que se disponían, lo cual, mejora la precisión en la estimación. Una vez que la metodología fue corregida, el procedimiento fue aplicado a las otras tres carreteras.

Por otro lado, el HSM (AASHTO, 2010) sugiere que se tengan entre 30-50 sitios de medición, lo cual, no pudo ser cumplido en 8 de los 11 tramos suburbanos, debido a que no habían más sitios disponibles en esas carreteras, tomándose toda la población y no una muestra, lo cual es representativo del lugar. El HSM (AASHTO, 2010) recomienda que la longitud mínima de cada segmentos analizado sea aproximadamente $161 \mathrm{~m}(0,10$ millas $)$, no obstante, en esta investigación hubieron pocos segmentos que no alcanzaron esa longitud, esto se debe a que se tomaron como segmentos a las rectas y curvas horizontales por separado, y algunas de ellas tuvieron menos de $161 \mathrm{~m}$, lo cual, puede mejorar la precisión del modelo, dado que un segmento incluye un solo elemento geométrico. También el HSM (AASHTO, 2010) sugiere que el número 
mínimo de accidentes en cada segmento analizado sea de 100 , lo que no se fue posible obtener en esta investigación, dado que sólo se contabilizaron los accidentes registrados por la Policía Nacional. Esto puede afectar levemente a la estimación de los factores de calibración.

Una de las limitaciones principales del estudio es que existen muchos accidentes de tránsito que no se registran por diversas causas, tales como accidentes leves en donde el dueño llama al mecánico, sin pasar por un informe de la policía; o cuando ha existido un accidente entre dos vehículos y los propietarios realizan un acuerdo extrajudicial, tampoco es reportado. En ese escenario, los accidentes reportados son menores a los accidentes reales (reportados y no reportados), por lo cual, los valores de factores de calibración tenderán a ser mayores. No obstante, ante la falta de datos no reportados, este estudio sólo trabajó con estadísticas de fuentes oficiales. A pesar de estas limitaciones, el presente estudio usó la información que tenía a disposición, la que posiblemente sea similar a otros lugares del país e incluso en otros sitios de otros países, por lo tanto, servirá como un estudio de comparación. Cabe mencionar que el estudio fue realizado en topografía montañosa o escarpada, en donde las carreteras suelen tener un mayor número de accidentes de tránsito, lo cual también se constituye un aporte al estado del arte.

\section{Conclusión}

El objetivo de este artículo fue estimar el factor de calibración del módulo de predicción de accidentes del HSM (AASHTO, 2010) en carreteras principales del cantón Loja. Estos factores servirán predecir el número de accidentes en estas carreteras o en carreteras con similares características geométricas, de entorno y operación. Luego del análisis de los resultados de esta investigación, se plantearon las siguientes conclusiones:

Aunque en los tramos rurales se tuvieron más accidentes observados que en los tramos suburbanos, en general, éstos tuvieron menores valores de factores de calibración que los de los tramos suburbanos. Esto posiblemente se deba a que los tramos rurales fueron más largos que los tramos suburbanos o que a las variables consideradas en cada modelo. En este caso, el modelo predictivo sobreestima el número de accidentes reales en carreteras rurales de dos carriles, mientras que, en carreteras suburbanas el modelo, en algunos casos, sobreestima y en otros subestima. El modelo de predicción de accidentes del HSM (AASHTO, 2010) es una herramienta muy útil al momento de justificar un proyecto nuevo de carreteras o un rediseño, ya que la alternativa de diseño que presente el menor número de accidentes, también presentará menores costos de operación. Sin embargo, este modelo debe ser previamente calibrado para las condiciones locales con el fin de obtener estimaciones más precisas.

\section{Agradecimientos}

Los autores agradecen a la Secretaría Nacional de Educación Superior, Ciencia, Tecnología e Innovación (SENESCYT) de la República del Ecuador y a la Universidad Técnica Particular de Loja por la ayuda otorgada para el desarrollo de esta investigación.

\section{Bibliografía}

AASHTO. (2010). Highway Safety Manual (Primera). Washington, EE. UU. Autodesk. (2015). AutoCAD Civil 3D. Retrieved from https://www.autodesk.com/education/freesoftware/autocad 
Berardo, M. G. (2015). Aplicación del modelo de predicción de accidentes viales del HSM (2010) en camino rural de dos carriles en Brasil. In Departamento de Construcciones Civiles, Facultad de Ciencias Exactas, Físicas y Naturales, Universidad Nacional de Córdoba (Vol. 2).

Berardo, M. G., Freire, R. G., Marchesini, P., Tartabini, M., \& Vanoli, G. (2013). Ajuste de Parámetros en Modelo de Predicción de Accidentes Viales del HSM (2010) para Argentina. In 9no Congreso de la Vialidad Uruguaya (pp. 1-23).

Bing. (2015). Mapa de las vías del cantón Loja. Retrieved from https://www.bing.com/maps

García, Y., \& Altamira, A. (2012a). Calibración del módulo de accidentes del Highway Safety Manual (HSM). In XVI Congreso Argentino de Vialidad y Tránsito. Córdoba, Argentina.

García, Y., \& Altamira, A. (2012b). Calibración del módulo de accidentes del Interactive Highway Safety Design Model (IHSDM) Caso de aplicación a San Juan Argentina. In III Congreso Iberroamericano de Seguridad Vial. Bogotá, Colombia.

Google. (2015). Street View Panamericana Norte. Retrieved from https://www.google.com.ec/maps

Lubliner, H. (2011). Evaluation of the Highway Safety Manual Crash Prediction Model for Rural TwoLane Highway Segments in Kansas. University of Kansas. Retrieved from https://kuscholarworks.ku.edu/handle/1808/8777? show=full

Martinelli, F., La Torre, F., \& Vadi, P. (2009). Calibration of the Highway Safety Manual's Accident Prediction Model for Italian Secondary Road Network. Transportation Research Record: Journal of the Transportation Research Board, 2103(2), 1-9. http://doi.org/https://doi.org/10.3141/2103-01

MTOP. (2012). Programa de inversión “Mantenimiento por nivel de servicio.” Quito. Retrieved from http://www.obraspublicas.gob.ec/wp-content/uploads/downloads/2015/12/ConservacionNiveles-De-Servicio.pdf

MTOP, \& Asociación del Sur. (2015). Estudios para la ampliación a cuatro carriles de la carretera Loja-Catamayo incluye el acceso al aeropuerto, ubicado en la provincia de Loja. Loja, Ecuador. Quito.

Ortiz, A. (2017). Estudio y Sistematización de diferentes variables de tráfico para la visualización a traves de un geovisor en las vías de acceso a la ciudad: Loja-Catamayo y Loja-Cuenca. Universidad Técnica Particular de Loja. Retrieved from http://dspace.utpl.edu.ec/handle/123456789/17068

Policía Nacional. (2017). Siniestros ANT 2014-2015-2016.

Sun, C., Brown, H., Edara, P., Carlos, B., \& Nam, K. (2013). Calibration of the Highway Safety Manual for Missouri. Missouri. Retrieved from https://library.modot.mo.gov/rdt/reports/tr201302/cmr14-007_reduced.pdf

Williamson, M., \& H., Z. (2012). Develop Calibration Factors for Crash Prediction Models for Rural Two-Lane Roadways in Illinois. Procedia - Social and Behavioral Sciences, 43, 330-338.

Xie, F., Gladhill, K., Dixon, K., \& Monsere, C. (2011). Calibrating the Highway Safety Manual Predictive Models for Oregon State Highways. Transportation Research Record: Journal of the Transportation Research Board, 2241, 19-28. http://doi.org/https://doi.org/10.3141/224103

Zúñiga, B. (2017). Estudio de las diferentes variables de tráfico vehicular en las vías Loja-Malacatos y Loja - Zamora para la visualización de información en un geovisor. Universidad Técnica Particular de Loja. Retrieved from http://dspace.utpl.edu.ec/handle/123456789/17069 